\title{
ANALYSIS OF REGULARIZATION METHODS FOR THE SOLUTION OF ILL-POSED PROBLEMS INVOLVING DISCONTINUOUS OPERATORS
}

\author{
F. FRÜHAUF, O. SCHERZER ${ }^{\ddagger}$ AND A. LEITÃO $\S$
}

\begin{abstract}
We consider a regularization concept for the solution of ill-posed operator equations, where the operator is composed of a continuous and a discontinuous operator. A particular application is level set regularization, where we develop a novel concept of minimizers. The proposed level set regularization is capable of handling changing topologies. A functional analytic framework explaining the splitting of topologies is given. The asymptotic limit of the level set regularization method is an evolution process, which is implemented numerically and the quality of the proposed algorithm is demonstrated by solving an inverse source problem.
\end{abstract}

Key words. Ill-Posed Problems, Regularization Methods, Level Set Methods

AMS subject classifications. 65J20, 47A52

1. Introduction. The goal of this paper is to analyze regularization models for the stable solution of ill-posed operator equations

$$
F(P(\phi))=y .
$$

Here $F$ is a continuous operator between Banach spaces $X$ and $Y$ and $P$ is a probably discontinuous operator from an admissible class $\mathcal{P}$ into $X$. Classical results on convergence and stability of variational regularization principles for solving non-linear ill-posed problems (see e.g. [20, 21, 11) in a Hilbert spaces setting such as

1. existence of a regularized solution,

2. stability of the regularized approximations,

3. approximation properties of the regularized solutions

are applicable if the operator $P$ is

1. bounded and linear or

2. nonlinear, continuous, and weakly closed.

In this paper we particularly emphasize on operator equations 1.1 where the operator $P$ is discontinuous. Of particular interest for this paper is

$$
P(t):=\left\{\begin{array}{lll}
0 & \text { for } \quad t<0 \\
1 & \text { for } \quad t \geq 0
\end{array}\right.
$$

With $P$ there is associated the admissible class

$$
\mathcal{P}:=\left\{u: u=\chi_{D} \text { where } D \subseteq \Omega \text { is measurable and } \mathcal{H}^{n-1}(\partial D)<\infty\right\} .
$$

Here

1. $\mathcal{H}^{n-1}(\partial D)$ denotes the $n$-1-dimensional Hausdorff-measure of the boundary of $D$;

2. $\chi_{D}$ denotes the characteristic function of the set $D$.

\footnotetext{
${ }^{\ddagger}$ Department of Computer Science, Universität Innsbruck, Technikerstraße 25, A-6020 Innsbruck, Austria, \{Florian.Frühauf, Otmar. Scherzer\}@uibk.ac.at

$\S$ Radon Institute for Comput. Appl. Math., Austrian Academy of Sciences, c/o Kepler Universität Linz, A-4040 Linz, Austria, Antonio.Leitao@oeaw.ac.at
} 
We call a regularization approach involving this projection level set regularization since we recover the boundary of an object $\partial D$, which is a level set (for instance with value 0 ) of a function $\phi$. The idea of considering characteristic functions as level sets of higher dimensional data has been used before in the context of multiphase flow (see e.g. [19, 27, 9]) and segmentation (see e.g. 8). Level set method have been used successively in many applications since the pioneering work of Osher \& Sethian [23. For solving inverse problems applications with level sets we refer to Santosa [25] and Burger [6].

In this work we base our considerations on ideas from nonlinear convex semigroup theory (cf. Brezis [5]) which allows to characterize the solution of an evolution process by implicit time steps of regularization models. Since our regularization models appear to be nonconvex, the theoretical results of nonlinear semigroup theory are not available. Simulating this approach, we show in this work that iterated regularization is well-posed, and (aside form the lack of theoretical results) we can interpret the iterated regularized solutions as time instance of an evolution process.

Various other models fit in the general framework of this paper but are not particularly emphasized: For instance for $a \in \mathbb{R}$ let us consider the following projection operator

$$
P^{a}(t):=\left\{\begin{aligned}
-a & \text { for } \quad t<-a \\
t & \text { for } \quad-a \leq t \leq a \\
a & \text { for } \quad t>a
\end{aligned}\right.
$$

with the admissible class

$$
\mathcal{P}_{a}:=\left\{u: u=P^{a}(\phi) \text { with } \phi \in H^{1}(\Omega)\right\} .
$$

The operator $P^{a}$ ensures that the recovered functions are absolutely bounded by $a$.

$$
P^{+}(t):=\exp (t)
$$

with the admissible class

$$
\mathcal{P}_{+}:=\left\{u: 0<u=P^{+}(\phi)\right\}
$$

can be used to guarantee non-negativity. Depending of the operator $P$ we actually solve a constraint optimization problem. With $P_{+}, P_{a}, P$ we guarantee that the solution is in the according admissible class.

The outline of this paper is as follows: In Section 2 we introduce the concept of level set regularization, based on considerations in 25, 6, 18. The level set regularization functionals derived in 18 are modified such that a convergence analysis becomes tractable (cf. Section 2.1). That is we show that each implicit time step is welldefined. This a prerequisite step in showing that the according evolution process is well-defined. To this end, we introduce a novel concept of a minimizer of regularization functionals involving discontinuous operators (cf. Section 2.2). A convergence analysis is presented in Section 2.3. The problem of numerical minimization is discussed in Section 3 and finally numerical examples are presented in Section 5. 
2. Analysis of Level Set Regularization. In the following we pose the general assumptions which we assume to hold all along this paper:

1. $\Omega \subseteq \mathbb{R}^{n}$ is bounded with $\partial \Omega$ piecewise $C^{1}$ (see e.g. [2]).

2. The operator $F: L^{1}(\Omega) \rightarrow Y$ is continuous and Fréchet-differentiable. $Y$ is a Banach space.

3. $\varepsilon, \alpha, \beta$ denote positive parameters.

4. We use the following notation:

(i) $\rightarrow$ denotes strong convergence,

(ii) $\stackrel{(*)}{\rightarrow}$ denotes weak $\left(^{*}\right)$ convergence,

(iii) $L^{p}(\Omega)$ denotes the space of measurable, $p$-times integrable functions,

(iv) $W^{1, p}(\Omega)$ denotes the Sobolev space of one time weakly differentiable functions where the function and its derivative are in $L^{p}$; in particular we set $H^{1}=W^{1,2}$.

(v) $\mathrm{BV}(\Omega)$ denotes the space of functions of bounded variation.

5. We assume that (1.1) has a solution, i.e. there exists a $z \in \mathcal{P}$ satisfying $F(z)=y$ and a function $\phi \in H^{1}(\Omega)$ satisfying $|\nabla \phi| \neq 0$ in a neighborhood of $\{\phi=0\}$ and $P(\phi)=z$. If $z=\chi_{A}$ and $\emptyset \neq A$, then we let

$$
\phi=-d_{\bar{A}}+d_{\overline{C A}}
$$

where $d_{\bar{A}}$ and $d_{\overline{C A}}$ denote the distance functions from $\bar{A}$, and $\overline{C A}$, respectively. Since $d_{\bar{A}}$ and $d_{\overline{C A}}$ are uniformly Lipschitz continuous (see e.g. [10]), they are in $L^{\infty}(\Omega)$. Moreover, $\left|\nabla d_{\bar{A}}\right| \leq 1$ and $\left|\nabla d_{\overline{C A}}\right| \leq 1$ (see again e.g. [10]). In particular this shows that $d_{\bar{A}}, d_{\overline{C A}} \in W^{1, \infty}(\Omega) \subseteq H^{1}(\Omega)$. Thus $z \in \mathcal{P}$ if $z=\chi_{A}$ and $A$ satisfies that the closure of the interior of $A$ is the closure of $A$.

We consider the unconstrained inverse problem of solving (1.1) with

$$
\begin{aligned}
P: H^{1}(\Omega) & \rightarrow \mathcal{P} . \\
\phi & \mapsto \frac{1}{2}+\frac{1}{2} \operatorname{sgn}(\phi)=: \frac{1}{2}+\frac{1}{2}\left\{\begin{array}{r}
1 \text { for } \phi \geq 0 \\
-1 \text { for } \phi<0
\end{array}\right.
\end{aligned}
$$

The standard form of Tikhonov regularization for solving (1.1) consists in minimizing the functional

$$
\mathcal{F}_{\alpha}(\phi):=\left\|F(P(\phi))-y^{\delta}\right\|_{Y}^{2}+\alpha\left\|\phi-\phi_{0}\right\|_{H^{1}(\Omega)}^{2}
$$

over $H^{1}(\Omega)$. Actually, we understand the minimizer $\phi_{\alpha}$ of this functional as

$$
\phi_{\alpha}=\lim _{\varepsilon \rightarrow 0+} \phi_{\varepsilon, \alpha},
$$

where the limit is understood in an appropriate sense (weak, weak* convergence) and $\phi_{\varepsilon, \alpha}$ minimizes the functional over $H^{1}(\Omega)$

$$
\mathcal{F}_{\varepsilon, \alpha}(\phi):=\left\|F\left(P_{\varepsilon}(\phi)\right)-y^{\delta}\right\|_{Y}^{2}+\alpha\left\|\phi-\phi_{0}\right\|_{H^{1}(\Omega)}^{2},
$$

where we use

$$
P_{\varepsilon}(\phi):=\left\{\begin{array}{rll}
0 & \text { for } \quad \phi<-\varepsilon, \\
1+\frac{\phi}{\varepsilon} & \text { for } \quad \phi \in[-\varepsilon, 0], \\
1 & \text { for } \quad \phi>0,
\end{array}\right.
$$

for approximating $P$ as $\varepsilon \rightarrow 0^{+}$. In this case we define

$$
P^{\prime}(t):=\lim _{\varepsilon \rightarrow 0+} P_{\varepsilon}^{\prime}(t)=\delta(t) .
$$


Here and in the following $\delta(t)$ denotes the one-dimensional $\delta$-distribution.

Taking into account that

$$
\left\|P_{\varepsilon}\left(\phi_{k}\right)-P_{\varepsilon}(\phi)\right\|_{L^{1}(\Omega)} \leq \frac{1}{\varepsilon} \operatorname{meas}(\Omega)\left\|\phi_{k}-\phi\right\|_{L^{2}(\Omega)},
$$

the proof of existence of a minimizer of the functional $\mathcal{F}_{\varepsilon, \alpha}$ is similar to the proof of existence of regularized solutions of Tikhonov functionals for approximately minimizing nonlinear ill-posed problems in [12, 26] (see also [11]).

TheOREM 2.1. For any $\phi_{0} \in H^{1}(\Omega)$ the functional $\mathcal{F}_{\varepsilon, \alpha}(c f .2 .2$ ) attains a minimizer $\phi_{\varepsilon, \alpha}$ in $H^{1}(\Omega)$.

2.1. Towards an Analysis of Level Set Regularization Techniques. In the following we outline the difficulties in performing a rigorous analysis for the functional $\mathcal{F}_{\alpha}$, defined in 2.1.

1. $\phi_{\varepsilon, \alpha}$ satisfies,

$$
\left\|P\left(\phi_{\varepsilon, \alpha}\right)\right\|_{L^{\infty}} \leq 1 \text { and }\left\|\phi_{\varepsilon, \alpha}-\phi_{0}\right\|_{H^{1}(\Omega)}<\infty .
$$

Since $L^{\infty}(\Omega)$ is the dual of $L^{1}(\Omega)$, i.e., $L^{1 *}(\Omega)=L^{\infty}(\Omega)$, we find that there exists a subsequence $\left\{\phi_{\varepsilon_{k}, \alpha_{k}}\right\}_{k \in \mathbb{N}}$ such that

$$
\phi_{\varepsilon_{k}, \alpha_{k}} \rightarrow \phi \text { in } H^{1}(\Omega) \text { and } P\left(\phi_{\varepsilon_{k}, \alpha_{k}}\right) \stackrel{*}{\rightarrow} z \text { in } L^{\infty}(\Omega) .
$$

There is no analytical evidence for $z \in \mathcal{P}$, i.e. it may not be in the range of the operator $P$.

2. To overcome this difficulty let us assume that the sequence $\left\{\phi_{\varepsilon_{k}, \alpha_{k}}\right\}_{k \in \mathbb{N}}$ satisfies that the Hausdorff measure of the boundary of the set

$$
\left\{x: \phi_{\varepsilon_{k}, \alpha_{k}}(x) \geq 0\right\}
$$

is uniformly bounded. Then the bounded variation semi-norm of $P\left(\phi_{\varepsilon_{k}, \alpha_{k}}\right)$ is uniformly bounded, and consequently $P\left(\phi_{\varepsilon_{k}, \alpha_{k}}\right)$ has a convergent subsequence in $L^{1}(\Omega)$ showing that $z$ is admissible.

This suggests to incorporate in the functional 2.1) as an additional regularization term the bounded variation semi-norm of $P(\phi)$, penalizing the length of the zero level set of $\phi$. Actually in design problems the necessity of incorporating such a term is well documented in [15, 16, 17]. This leads to the following modified regularization method of minimizing

$$
\mathcal{G}_{\alpha}(\phi):=\left\|F(P(\phi))-y^{\delta}\right\|_{Y}^{2}+2 \beta \alpha|P(\phi)|_{\mathrm{BV}}+\alpha\left\|\phi-\phi_{0}\right\|_{H^{1}(\Omega)}^{2} .
$$

In order to guarantee existence of a minimizer of $\mathcal{G}_{\alpha}$ we introduce a novel concept of a minimizer:

\subsection{Minimizing Concept.}

DeFinition 2.2 .

1. A pair of functions

$$
(z, \phi) \in L^{\infty}(\Omega) \times H^{1}(\Omega)
$$

is called admissible 
(i) if there exists a sequence $\left\{\phi_{k}\right\}_{k \in \mathbb{N}}$ in $H^{1}(\Omega)$ such that $\phi_{k} \rightarrow \phi$ with respect to the $L^{2}(\Omega)$-norm and

(ii) if there exists a sequence $\left\{\varepsilon_{k}\right\}_{k \in \mathbb{N}}$ of positive numbers converging to zero such that

$$
P_{\varepsilon_{k}}\left(\phi_{k}\right) \rightarrow z \text { in } L^{1}(\Omega) .
$$

2. A minimizer of $\mathcal{G}_{\alpha}$ is considered any admissible pair of functions $(z, \phi)$ minimizing

$$
\mathcal{G}_{\alpha}(z, \phi)=\left\|F(z)-y^{\delta}\right\|_{Y}^{2}+\alpha \rho(z, \phi)
$$

over all admissible pairs. Here

$$
\rho(z, \phi):=\inf \liminf _{k \rightarrow \infty}\left\{2 \beta\left|P_{\varepsilon_{k}}\left(\phi_{k}\right)\right|_{\mathrm{BV}}+\left\|\phi_{k}-\phi_{0}\right\|_{H^{1}(\Omega)}^{2}\right\}
$$

where the infimum is taken with respect to all sequences $\left\{\varepsilon_{k}\right\}_{k \in \mathbb{N}}$ satisfying Item 1(ii) and $\left\{\phi_{k}\right\}_{k \in \mathbb{N}}$ satisfying Item $1(i)$.

A generalized minimizer of $\mathcal{G}_{\alpha}(\phi)$ is a minimizer of $\mathcal{G}_{\alpha}(z, \phi)$ on the set of admissible pairs.

The following lemma is to show that the functional $\rho$ is coercive on the set of admissible pairs.

Lemma 2.3. For each $(z, \phi)$ admissible

$$
2 \beta|z|_{\mathrm{BV}}+\left\|\phi-\phi_{0}\right\|_{H^{1}(\Omega)}^{2} \leq \rho(z, \phi) .
$$

Proof. Let $(z, \phi)$ be an admissible pair, then there exists sequences $\left\{\varepsilon_{k}\right\}_{k \in \mathbb{N}}$ and $\left\{\phi_{k}\right\}_{k \in \mathbb{N}}$ satisfying Items 1(i) and 1(ii) and

$$
\rho(z, \phi)=\lim _{k \rightarrow \infty} 2 \beta\left|P_{\varepsilon_{k}}\left(\phi_{k}\right)\right|_{\mathrm{BV}}+\left\|\phi_{k}-\phi_{0}\right\|_{H^{1}(\Omega)}^{2} .
$$

By the weak lower semi-continuity of the BV and $H^{1}$-norms it follows that

$$
\begin{aligned}
\left\|\phi-\phi_{0}\right\|_{H^{1}(\Omega)}^{2} & \leq \liminf _{k \in \mathbb{N}}\left\|\phi_{k}-\phi_{0}\right\|_{H^{1}(\Omega)}^{2} \\
|z|_{\mathrm{BV}} & \leq \liminf _{k \in \mathbb{N}}\left|P_{\varepsilon_{k}}\left(\phi_{k}\right)\right|_{\mathrm{BV}}
\end{aligned}
$$

which proves the assertion.

The definition of $\rho(z, \phi)$ is impractical, since it is defined via a relaxation procedure. The following arguments allow an explicit characterization of this functional. From several experiments which we outline below, we conjecture the following characterization of the functional $\rho(z, \phi)$.

Conjecture 2.4. We denote by

$$
\Phi_{+}=\{x \in \Omega: \phi(x)>0\} \text { and } \Phi_{-}=\{x \in \Omega: \phi(x)<0\}
$$

and

$$
C \Phi=\Omega \backslash\left(\Phi_{+} \cup \Phi_{-}\right) .
$$


(i) If $\partial \Phi_{+} \cap \Omega=\partial \Phi_{-} \cap \Omega$, then

$$
\begin{aligned}
\rho(z, \phi) & =2 \beta \mathcal{H}^{n-1}\left(\partial \Phi_{-} \cap \Omega\right)+\left\|\phi-\phi_{0}\right\|_{H^{1}(\Omega)}^{2} \\
& =2 \beta \mathcal{H}^{n-1}\left(\partial \Phi_{+} \cap \Omega\right)+\left\|\phi-\phi_{0}\right\|_{H^{1}(\Omega)}^{2} .
\end{aligned}
$$

(ii) If the $n$-dimensional Lebesgue measure $\lambda^{n}(C \Phi)>0$, then $z$ is not unique identified, in particular $z$ can attain all values in $[0,1]$ in $C \Phi$. We conjecture, that

$$
\inf _{z \text { admissible }} \rho(z, \phi)=2 \beta \mathcal{H}^{n-1}(S)+\left\|\phi-\phi_{0}\right\|_{H^{1}(\Omega)}^{2} .
$$

The problem consists in finding the surface $S$ of minimal n-1-dimensional Hausdorff measure, which is contained in $C \Phi$ and divides $\Omega$ in two sets. One set completely contains $\Phi_{+}$and the other set contains $\Phi_{-}$, (cf. Figures 2.1 and 2.2).

Intuitively the conjecture is quite obvious. Assuming the conjecture to be true we are further led to conjecture that the functional $\rho$ is independent of the choice of the approximation $P_{\varepsilon}$. Thus any other approximation of $P$ with Lipschitz-continuous functions $P_{\varepsilon}$ approximating the $\delta$-distribution is suitable as well.
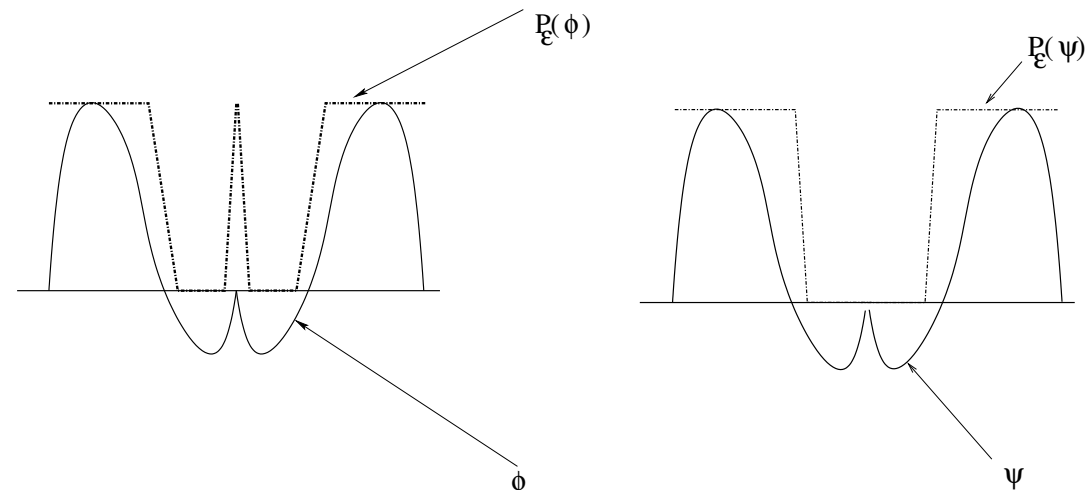

FIG. 2.1. $n=1$ : The functions $\phi$ and $P_{\varepsilon}(\phi)$ (left): $\left|P_{\varepsilon}(\phi)\right|_{\mathrm{BV}}=4$. A slight perturbation: $\psi$ and $P_{\varepsilon}(\psi)$ (right): $\left|P_{\varepsilon}(\psi)\right|_{\mathrm{BV}}=2$.
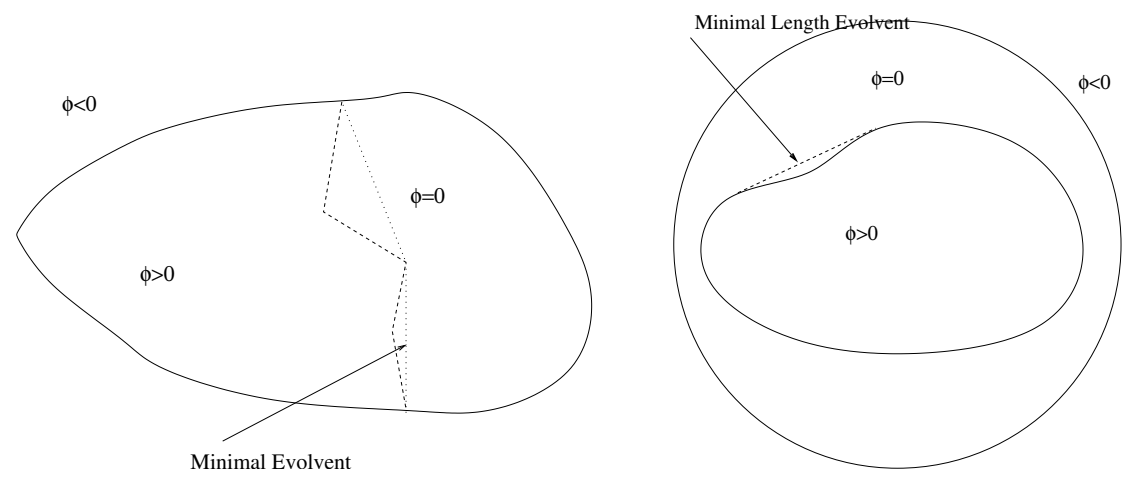

FIG. 2.2. The minimal evolvent in $C \Phi$

REMARK 2.5. For $\phi \in H^{1}(\Omega)$ where $\{\phi=0\}$ is a set of positive Lebesgue measure (cf. Figure 2.3) it is possible to find sequences $\left\{\phi_{k}\right\}_{k \in \mathbb{N}}$ and $\left\{\tilde{\phi}_{k}\right\}_{k \in \mathbb{N}}$, which converge 
strongly to $\phi$ in $L^{2}(\Omega)$, respectively. But the limits of the projections are different, i.e., $z=\lim _{k \rightarrow \infty} P_{\varepsilon_{k}}\left(\phi_{k}\right) \neq \tilde{z}=\lim _{k \rightarrow \infty} P_{\varepsilon_{k}}\left(\tilde{\phi}_{k}\right)$, cf. Figure 2.3. In such a situation we have $\rho(z, \phi) \neq \rho(\tilde{z}, \phi)$.

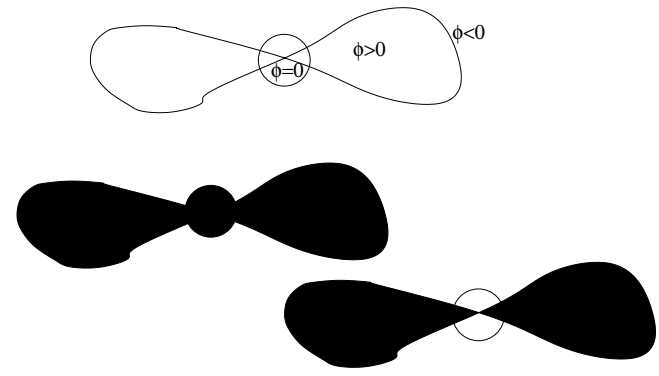

FIG. 2.3. Top: The level set function has critical values (i.e. $|\nabla \phi|=0$ in a circle). Bottom: Two possible functions $z$ and $\tilde{z}$. The black value corresponds to a value of $z=1$.

In the following we summarize some properties of the functional $\rho$.

LEMma 2.6. The functional $\rho$ satisfies

$$
\rho(z, \phi) \leq \liminf _{n \in \mathbb{N}} \rho\left(z_{n}, \phi_{n}\right)
$$

if $z_{n} \rightarrow z$ in $L^{1}(\Omega)$ and if $\phi_{n} \rightarrow \phi$ in $H^{1}(\Omega)$ and $\left(z_{n}, \phi_{n}\right)$ is admissible.

Proof. ¿From the definition of the functional $\rho$ and Lemma 2.3 it follows that the functional $\rho$ is a $\Gamma^{-}$-limit (see e.g. [3]) and thus it is weak lower semi-continuous. 口

REMARK 2.7. Suppose for the moment that $P$ is a continuous operator, in which case we can set $P_{\varepsilon}:=P$. Then the admissible class is just the set of pairs $(z, \phi)$ satisfying $P(\phi)=z$. This is just another formulation of constraint optimization. In our context $P$ is discontinuous and therefore we consider the more general concept of admissible pairs.

EXAMPLE 2.8. Let $\phi \in H^{1}(\Omega)$ satisfying $|\nabla \phi|>0$ in a neighborhood of $\{\phi=0\}$.

1. Let $\phi_{k}=\phi \in H^{1}(\Omega)$ and let $z=P\left(\phi_{k}\right)$. Since for any sequence $\varepsilon_{k} \rightarrow 0$

$$
P_{\varepsilon_{k}}\left(\phi_{k}\right) \rightarrow z \text { in } L^{1}(\Omega),
$$

it follows that $(z, \phi)$ is admissible.

2. Let $\phi \in H^{1}(\Omega)$ and denote by $\phi_{k}=\frac{1}{k} \phi$. Then there is a sequence $\varepsilon_{k} \rightarrow 0$ with

$$
P_{\varepsilon_{k}}\left(\phi_{k}\right) \rightarrow z \text { in } L^{1}(\Omega) .
$$

Consequently, $(z, 0)$ is admissible.

The consequence of the second item is striking. Suppose that $\phi_{0}=0$ and that there exists a minimizer $\phi_{\alpha} \neq 0$ of $(2.3)$. Then for any $k \in \mathbb{N}$

$$
\mathcal{G}_{\alpha}\left(\phi_{\alpha} / k\right)<\mathcal{G}_{\alpha}(\phi)
$$

showing that a minimizer of $\mathcal{G}_{\alpha}$ is not attained in a common setting. However, the pair $\left(z=P\left(\phi_{\alpha}\right), 0\right)$ is admissible and can be considered as the generalized solution.

Note that in this example we consider only functions $\phi \in H^{1}(\Omega)$ without critical points along the zero level set. 
2.3. Well-Posedness and Convergence Analysis.

Theorem 2.9 (Well-Posedness). Both the functional $\mathcal{G}_{\alpha}$ and the functional

$$
\tilde{\mathcal{G}_{\alpha}}(z, \phi):=\left\|F(z)-y^{\delta}\right\|_{Y}^{2}+2 \beta \alpha|z|_{\mathrm{BV}}+\alpha\left\|\phi-\phi_{0}\right\|_{H^{1}(\Omega)}^{2}
$$

attain minimizers on the set of admissible pairs.

Proof.

1. Since $(0,0)$ is admissible, the set of admissible pairs is not empty.

2. Suppose that $\left\{\left(z_{k}, \phi_{k}\right)\right\}_{k \in \mathbb{N}}$ is a sequence of admissible pairs such that

$$
\mathcal{G}_{\alpha}\left(z_{k}, \phi_{k}\right) \rightarrow \inf \mathcal{G}_{\alpha} \leq \mathcal{G}_{\alpha}(0,0)<\infty .
$$

From Lemma 2.3 it follows that $\left\{\left(z_{k}, \phi_{k}\right)\right\}_{k \in \mathbb{N}}$ is uniformly bounded in $\mathrm{BV} \times H^{1}(\Omega)$. By the Sobolev embedding theorem there exists a subsequence, denoted again by $\left\{\phi_{k}\right\}_{k \in \mathbb{N}}$ such that

$$
\begin{array}{r}
\phi_{k} \rightarrow \phi \text { in } H^{1}(\Omega) \text { and } \phi_{k} \rightarrow \phi \text { in } L^{2}(\Omega), \text { and } \\
z_{k} \rightarrow z \text { in } L^{1}(\Omega), 2 \beta|z|_{\mathrm{BV}} \leq \rho(z, \phi) \leq \liminf _{k \rightarrow \infty} \rho\left(z_{k}, \phi_{k}\right) .
\end{array}
$$

Since $\rho$ is weakly lower semi-continuous (cf. Lemma 2.6) it follows that

$$
\begin{aligned}
\inf \mathcal{G}_{\alpha} & =\lim _{k \rightarrow \infty} \mathcal{G}_{\alpha}\left(z_{k}, \phi_{k}\right) \\
& =\lim _{k \rightarrow \infty}\left\{\left\|F\left(z_{k}\right)-y^{\delta}\right\|_{Y}^{2}+\alpha \rho\left(z_{k}, \phi_{k}\right)\right\} \\
& \geq\left\|F(z)-y^{\delta}\right\|_{Y}^{2}+\alpha \rho(z, \phi) \\
& =\mathcal{G}_{\alpha}(z, \phi) .
\end{aligned}
$$

3. It remains to prove that $(z, \phi)$ is admissible. For $k$ fixed; since $\left(z_{k}, \phi_{k}\right)$ is admissible there exists a sequence $\left\{\varepsilon_{k, l}\right\}_{l \in \mathbb{N}}$ of positive numbers and a sequence $\left\{\phi_{k, l}\right\}_{l \in \mathbb{N}}$ in $H^{1}(\Omega)$ such that

$$
\phi_{k, l} \rightarrow_{l \rightarrow \infty} \phi_{k} \text { in } L^{2}(\Omega), \quad P_{\varepsilon_{k, l}}\left(\phi_{k, l}\right) \rightarrow_{l \rightarrow \infty} z_{k} \text { in } L^{1}(\Omega) .
$$

Thus there exists an index $l(k) \in \mathbb{N}$ such that

(i) $\varepsilon_{k, l(k)}<\frac{1}{2} \varepsilon_{k-1, l(k-1)}$;

(ii) $\left\|\phi_{k, l(k)}-\phi_{k}\right\|_{L^{2}(\Omega)} \leq \frac{1}{k}$;

(iii) $\left\|P_{\varepsilon_{k, l(k)}}\left(\phi_{k, l(k)}\right)-z_{k}\right\|_{L^{1}(\Omega)} \leq \frac{1}{k}$.

$$
\psi_{k}:=\phi_{k, l(k)} \text { and } \eta_{k}:=\varepsilon_{k, l(k)} .
$$

Then, since

$$
\psi_{k} \rightarrow \phi \text { in } L^{2}(\Omega) \text { and } P_{\eta_{k}}\left(\psi_{k}\right) \rightarrow z \text { in } L^{1}(\Omega),
$$

we see that $(z, \phi)$ is admissible.

The proof of existence of a minimizer of $\tilde{\mathcal{G}_{\alpha}}$ is analogous as for $\mathcal{G}_{\alpha}$, and thus omitted.

We have shown that for any positive parameters $\alpha, \beta$ the functionals $\mathcal{G}_{\alpha}$ and $\tilde{\mathcal{G}_{\alpha}}$ both attain a minimizer. 
In the sequel we denote by $\left(z_{\alpha}, \phi_{\alpha}\right)$ a minimizer of $\mathcal{G}_{\alpha}$.

In the following we summarize some convergence result for the regularized minimizers, which are based on the existence of a minimum norm solution:

THEOREM 2.10 (Existence of a minimum norm solution). Under the general assumptions of this paper there exists a minimum norm solution $\left(z^{\dagger}, \phi^{\dagger}\right)$, that is an admissible pair of functions that satisfies

1. $F\left(z^{\dagger}\right)=y$,

2. $\rho\left(z^{\dagger}, \phi^{\dagger}\right)=m s:=\inf \{\rho(z, \phi):(z, \phi)$ admissible and $F(z)=y\}$.

Proof.

1. According to assumption 5 there exists a function $\tilde{z} \in \mathcal{P}$ and a function $\tilde{\phi} \in H^{1}(\Omega)$ such that $P(\tilde{\phi})=\tilde{z}$ and $F(\tilde{z})=y$. Then the pair $(\tilde{z}, \tilde{\phi})$ is admissible for the sequence $\tilde{\phi}_{k}=\tilde{\phi}$, because $P_{\varepsilon_{k}}\left(\tilde{\phi}_{k}\right) \rightarrow \tilde{z}$ converges in $L^{1}(\Omega)$ for every sequence $\varepsilon_{k} \rightarrow 0$ due to the fact that $P_{\varepsilon_{k}}$ is a convolution of $P$ with a $\delta$-distribution, i.e. $P_{\varepsilon_{k}}=P * \delta_{k}$. Thus the set of admissible pairs with $F(z)=y$ is not empty.

2. Suppose that $\left\{\left(z_{k}, \phi_{k}\right)\right\}_{k \in \mathbb{N}}$ is a sequence of admissible pairs with $F\left(z_{k}\right)=y$ such that

$$
\rho\left(z_{k}, \phi_{k}\right) \rightarrow \operatorname{ms} \leq \rho(\tilde{z}, \tilde{\phi})<\infty
$$

From Lemma 2.3 it follows that the sequences $\left\{\phi_{k}\right\}_{k \in \mathbb{N}}$ and $\left\{z_{k}\right\}_{k \in \mathbb{N}}$ are uniformly bounded in $H^{1}(\Omega)$ and $\operatorname{BV}(\Omega)$, respectively. Thus there exists subsequences, again denoted by $\left\{\phi_{k}\right\}_{k \in \mathbb{N}}$ and $\left\{z_{k}\right\}_{k \in \mathbb{N}}$, such that

$$
\phi_{k} \rightarrow \phi^{\dagger} \text { in } L^{2}(\Omega), z_{k} \rightarrow z^{\dagger} \text { in } L^{1}(\Omega) .
$$

Since $\rho$ is weakly lower semi-continuous, it follows

$$
\mathrm{ms}=\lim _{k \rightarrow \infty} \rho\left(z_{k}, \phi_{k}\right) \geq \rho\left(z^{\dagger}, \phi^{\dagger}\right) .
$$

Since $F$ is continuous on $L^{1}(\Omega), F\left(z^{\dagger}\right)=\lim _{k \rightarrow \infty} F\left(z_{k}\right)=y$. Analogously the proof of Theorem 2.9 it follows, that $\left(z^{\dagger}, \phi^{\dagger}\right)$ is admissible and therefore a minimal norm solution.

Below, we summarize a stability and convergence result. The proof uses classical techniques from the analysis of Tikhonov type regularization methods (e.g. see [12, 26, 1, 11, 22]) and thus is omitted:

THEOREM 2.11 .

Convergence: Let $\left\|y^{\delta}-y\right\|_{Y} \leq \delta$. If $\alpha=\alpha(\delta)$ satisfies

$$
\lim _{\delta \rightarrow 0} \alpha(\delta)=0 \text { and } \lim _{\delta \rightarrow 0} \frac{\delta^{2}}{\alpha(\delta)}=0 .
$$

Then, for a sequence $\left\{\delta_{k}\right\}_{k \in \mathbb{N}}$ converging to 0 there exists a sequence $\left\{\alpha_{k}:=\alpha\left(\delta_{k}\right)\right\}_{k \in \mathbb{N}}$ such that $\left(z_{\alpha_{k}}, \phi_{\alpha_{k}}\right)$ converges in $L^{1}(\Omega) \times L^{2}(\Omega)$ to a minimal norm solution.

3. Numerical Solution. We consider a stabilized functional

$$
\mathcal{G}_{\varepsilon, \alpha}(\phi):=\left\|F\left(P_{\varepsilon}(\phi)\right)-y^{\delta}\right\|_{Y}^{2}+2 \beta \alpha\left|P_{\varepsilon}(\phi)\right|_{\mathrm{BV}}+\alpha\left\|\phi-\phi_{0}\right\|_{H^{1}(\Omega)}^{2} .
$$

This functional is well-posed as the following lemma shows:

Lemma 3.1. For any $\phi_{0} \in H^{1}(\Omega)$ the functional 3.1) attains a minimizer. 
Proof. The proof is similar to the proof of Theorem 2.1 by taking into account that for any sequence $\left\{\phi_{k}\right\}_{k \in \mathbb{N}}$ converging weakly to $\phi$ in the $H^{1}(\Omega)$-norm, there exists a strongly convergent subsequence in $L^{2}(\Omega)$. Denoting the subsequence again by $\left\{\phi_{k}\right\}_{k \in \mathbb{N}}$ we find

1.

$$
\left\|P_{\varepsilon}\left(\phi_{k}\right)-P_{\varepsilon}(\phi)\right\|_{L^{1}(\Omega)} \leq \frac{1}{\varepsilon} \operatorname{meas}(\Omega)\left\|\phi_{k}-\phi\right\|_{L^{2}(\Omega)} \rightarrow 0 .
$$

2. Therefore

$$
\left|P_{\varepsilon}(\phi)\right|_{\mathrm{BV}} \leq \liminf _{k \rightarrow \infty}\left|P_{\varepsilon}\left(\phi_{k}\right)\right|_{\mathrm{Bv}}
$$

Now, the assertion can be proved analogously as Theorem 2.1 .

In the following we show that for $\varepsilon \rightarrow 0$ the minimizer of $\mathcal{G}_{\varepsilon, \alpha}$ approximates a minimizer of $\mathcal{G}_{\alpha}$, i.e., it approximates an admissible pair.

THEOREM 3.2. Let $\phi_{\varepsilon, \alpha}$ be a minimizer of $\mathcal{G}_{\varepsilon, \alpha}$. Then for $\varepsilon_{k} \rightarrow 0$, there exists a convergent subsequence $\left(P_{\varepsilon_{k}}\left(\phi_{\varepsilon_{k}, \alpha}\right), \phi_{\varepsilon_{k}, \alpha}\right) \rightarrow(\tilde{z}, \tilde{\phi})$ in $L^{1}(\Omega) \times L^{2}(\Omega)$, and the limit minimizes $\mathcal{G}_{\alpha}$ in the set of admissible pairs.

Proof.

1. The infimum of $\mathcal{G}_{\alpha}$ is attained (cf. Theorem 2.9), i.e., there exists $\left(z_{\alpha}, \phi_{\alpha}\right)$ minimizing $\mathcal{G}_{\alpha}$ over all admissible pairs. In particular, taking into account the definition of admissible pairs, there exists a sequence $\left\{\varepsilon_{k}\right\}_{k \in \mathbb{N}}$ of positive numbers converging to zero and a corresponding sequence $\left\{\phi_{k}\right\}_{k \in \mathbb{N}}$ in $H^{1}(\Omega)$ satisfying

$$
\begin{aligned}
\left(P_{\varepsilon_{k}}\left(\phi_{k}\right), \phi_{k}\right) & \rightarrow\left(z_{\alpha}, \phi_{\alpha}\right) \text { in } L^{1}(\Omega) \times L^{2}(\Omega), \\
\rho\left(z_{\alpha}, \phi_{\alpha}\right) & =\lim _{k \rightarrow \infty}\left\{2 \beta\left|P_{\varepsilon_{k}}\left(\phi_{k}\right)\right|_{\mathrm{BV}}+\left\|\phi_{k}-\phi_{0}\right\|_{H^{1}(\Omega)}^{2}\right\} .
\end{aligned}
$$

2. Let $\phi_{\varepsilon_{k}}$ be a minimizer of $\mathcal{G}_{\varepsilon_{k}, \alpha}$. The sequence $\left\{\phi_{\varepsilon_{k}}\right\}_{k \in \mathbb{N}}$ is uniformly bounded in $H^{1}(\Omega)$. Thus it has a weakly convergent subsequence (which is again denoted by the same indices) and the weak limit is denoted $\tilde{\phi}$. Moreover, $\left\{P_{\varepsilon_{k}}\left(\phi_{\varepsilon_{k}}\right)\right\}_{k \in \mathbb{N}}$ is uniformly bounded in $\operatorname{BV}(\Omega)$. Thus, by the compact Sobolev embedding theorem there exists a subsequence $\left\{\phi_{\varepsilon_{k}}\right\}_{k \in \mathbb{N}}$ (again denoted with the same indices) satisfying

$$
\phi_{\varepsilon_{k}} \rightarrow \tilde{\phi} \text { in } L^{2}(\Omega) \text {, and } P_{\varepsilon_{k}}\left(\phi_{\varepsilon_{k}}\right) \rightarrow \tilde{z} \text { in } L^{1}(\Omega) .
$$

Thus $(\tilde{z}, \tilde{\phi}) \in \mathcal{P} \times H^{1}(\Omega)$ is admissible.

3. From the definition of $\rho$ and the continuity of $F: L^{1}(\Omega) \rightarrow Y$ it follows that

$$
\begin{aligned}
\left\|F(\tilde{z})-y^{\delta}\right\|_{Y}^{2} & =\lim _{k \rightarrow \infty}\left\|F\left(P_{\varepsilon_{k}}\left(\phi_{\varepsilon_{k}}\right)\right)-y^{\delta}\right\|_{Y}^{2}, \\
\rho(\tilde{z}, \tilde{\phi}) & \leq \liminf _{k \rightarrow \infty}\left\{2 \beta\left|P_{\varepsilon_{k}}\left(\phi_{\varepsilon_{k}}\right)\right|_{\mathrm{BV}}+\left\|\phi_{\varepsilon_{k}}-\phi_{0}\right\|_{H^{1}(\Omega)}^{2}\right\}
\end{aligned}
$$

This shows that

$$
\begin{aligned}
\mathcal{G}_{\alpha}(\tilde{z}, \tilde{\phi}) & \leq \liminf _{k \rightarrow \infty} \mathcal{G}_{\varepsilon_{k}, \alpha}\left(\phi_{\varepsilon_{k}}\right) \\
& \leq \liminf _{k \rightarrow \infty} \mathcal{G}_{\varepsilon_{k}, \alpha}\left(\phi_{k}\right) \\
& =\left\|F\left(z_{\alpha}\right)-y^{\delta}\right\|_{Y}^{2}+\alpha \rho\left(z_{\alpha}, \phi_{\alpha}\right) \\
& =\inf \mathcal{G}_{\alpha} .
\end{aligned}
$$

Therefore the infimum of $\mathcal{G}_{\alpha}$ is attained at $(\tilde{z}, \tilde{\phi})$. 
Theorem 3.2 justifies to use the functionals $\mathcal{G}_{\varepsilon, \alpha}$ for approximation of the minimizer of $\mathcal{G}_{\alpha}$. In contrast to the minimizer of $\mathcal{G}_{\varepsilon, \alpha}$, which is a function in $H^{1}(\Omega)$, the minimizer of $\mathcal{G}_{\alpha}$ is an admissible pair $\left(z_{\alpha}, \phi_{\alpha}\right)$. Recall that the function $z_{\alpha}$ is not uniquely defined by $\phi_{\alpha}$ if it attains critical values in a neighborhood of the zero level set (cf. Remark 2.5).

For numerical purposes it is convenient to derive the optimality conditions of a minimizer of this functional. To this end we consider the functional $\mathcal{G}_{\varepsilon, \alpha}$ with $Y=L^{2}(\partial \Omega)$.

Since $P_{\varepsilon}^{\prime}(\phi)$ is self-adjoint, we can write the formal optimality condition for a minimizer of the functional $\mathcal{G}_{\varepsilon, \alpha}$ as follows:

$$
\alpha(\Delta-I)\left(\phi-\phi_{0}\right)=R_{\varepsilon, \alpha, \beta}(\phi),
$$

where

$$
R_{\varepsilon, \alpha, \beta}(\phi)=P_{\varepsilon}^{\prime}(\phi) F^{\prime}\left(P_{\varepsilon}(\phi)\right)^{*}\left(F\left(P_{\varepsilon}(\phi)\right)-y^{\delta}\right)-\beta \alpha P_{\varepsilon}^{\prime}(\phi) \nabla \cdot\left(\frac{\nabla P_{\varepsilon}(\phi)}{\left|\nabla P_{\varepsilon}(\phi)\right|}\right) .
$$

4. Iterative Regularization and the Relation to Dynamic Level Set Methods. For $n=1$ set $\mathcal{G}_{\alpha}^{(1)}(z, \phi)=\mathcal{G}_{\alpha}(z, \phi)$ (cf. 2.4). Iterative regularization consists in minimizing the family of functionals

$$
\mathcal{G}_{\alpha}^{(n)}(z, \phi)=\left\|F(z)-y^{\delta}\right\|_{Y}^{2}+\alpha \rho^{(n)}(z, \phi)
$$

where $\rho^{(n)}$ is the functional $\rho$ (as defined in 2.5) with $\phi_{0}$ replaced by $\phi_{n-1}$. The minimizer of $\mathcal{G}_{\alpha}^{(n)}(z, \phi)$ is denoted by $\phi_{n}$.

Proceeding as before, we find that $\phi_{n}$ can be realized by solving the formal optimality condition

$$
\alpha(\Delta-I)\left(\phi-\phi_{n-1}\right)=R_{\varepsilon, \alpha, \beta}(\phi) .
$$

Identifying $\alpha=1 / \Delta t, t_{n}=n \Delta t$, and $\phi_{n}=\phi\left(t_{n}\right), n=0,1, \ldots$ we find

$$
(\Delta-I)\left(\frac{\phi\left(t_{n}\right)-\phi\left(t_{n-1}\right)}{\Delta t}\right)=R_{\varepsilon, 1 / \Delta t, \beta}\left(\phi\left(t_{n}\right)\right) .
$$

Considering $\Delta t$ as a time discretization and using $\beta=b_{\Delta} \Delta t$ we find that in a formal sense the iterative regularized solution $\phi_{n}$ is a solution of an implicit time step for the dynamic system

$$
(\Delta-I)\left(\frac{\partial \phi(t)}{\partial t}\right)=R_{\varepsilon, 1 / \Delta t, b_{\Delta} \Delta t}(\phi(t)) .
$$

In our numerical experiments we have calculated the solution of the dynamic system (4.4).

For each time step it is required to solve equation 4.3$). \phi\left(t_{n}\right)$ in 4.3 can be solved with a fixed point iteration: setting $\phi\left(t_{n-1}\right)=\phi^{(0)}$, we get $\phi\left(t_{n}\right)=\lim _{k \rightarrow \infty} \phi^{(k)}$

$$
(\Delta-I)\left(\frac{\phi^{(k+1)}-\phi^{(0)}}{\Delta t}\right)=R_{\varepsilon, 1 / \Delta t, b_{\Delta} \Delta t}\left(\phi^{(k)}\right) .
$$

In our numerical experiments we observed that the iteration does not significantly improve after the first iteration (cf. Figure 4.1). This behavior becomes transparent 
by noting the $H^{1}$-seminorm typically dominates the $L^{2}$-norm in the quadratic regularization term. The $H^{1}$-seminorm difference of the regularized solution and $\phi^{(0)}$ is small if it is just shifted up or down. In numerical experiments it is observed that the first iteration almost corresponds to a horizontal shift of $\phi^{(0)}$ such that the residual functional is minimized (cf. Figure 4.2) and also the further iterations are again nearly horizontally shifted versions of $\phi^{(0)}$ (cf. Figure 4.3).

In almost all test examples the residual $\left\|F\left(P_{\varepsilon}\left(\phi^{(k)}\right)\right)-y^{\delta}\right\|^{2}$ is oscillating in dependence of $k$ (cf. Figure 4.2 ) and smallest for $k=1$.

The above consideration justify to restrict attention to the approximate solution of the dynamic system (4.2) where in each time step only one iteration step of 4.5) is used, i.e., we use an explicit Euler method for solving the evolution process. In this case numerical instabilities may occur by dividing by small absolute values of the gradient in the differential $\nabla \cdot\left(\frac{\nabla P_{\varepsilon}(\phi)}{\left|\nabla P_{\varepsilon}(\phi)\right|}\right)$. Thus, for numerical purpose it is convenient to introduce a small positive number $h$ and replace the differential by

$$
\nabla \cdot\left(\frac{\nabla P_{\varepsilon}(\phi)}{\sqrt{\left|\nabla P_{\varepsilon}(\phi)\right|^{2}+h^{2}}}\right) .
$$

Usually semi-implicit iteration schemes require a less restrictive time marching (this approach is commonly referred as Dziuk's method). The implementation would require to solve

$$
\begin{aligned}
(\Delta-I)\left(\frac{\phi^{(k+1)}-\phi^{(0)}}{\Delta t}\right)= & P_{\varepsilon}^{\prime}\left(\phi^{(k)}\right) F^{\prime}\left(P_{\varepsilon}\left(\phi^{(k)}\right)\right)^{*}\left(F\left(P_{\varepsilon}\left(\phi^{(k)}\right)\right)-y^{\delta}\right) \\
& -b_{\Delta} P_{\varepsilon}^{\prime}\left(\phi^{(k)}\right) \nabla \cdot\left(\frac{\nabla P_{\varepsilon}\left(\phi^{(k+1)}\right)}{\sqrt{\left|\nabla P_{\varepsilon}\left(\phi^{(k)}\right)\right|^{2}+h^{2}}}\right) .
\end{aligned}
$$

In implementation of this approach the difficulty arises that the function in front of $\nabla \cdot\left(\nabla P_{\varepsilon}\left(\phi^{(k+1)}\right) / \sqrt{\left|\nabla P_{\varepsilon}\left(\phi^{(k)}\right)\right|^{2}+h^{2}}\right)$ vanishes outside of a neighborhood of the zero level set, which makes it almost impossible to implement this scheme efficiently.
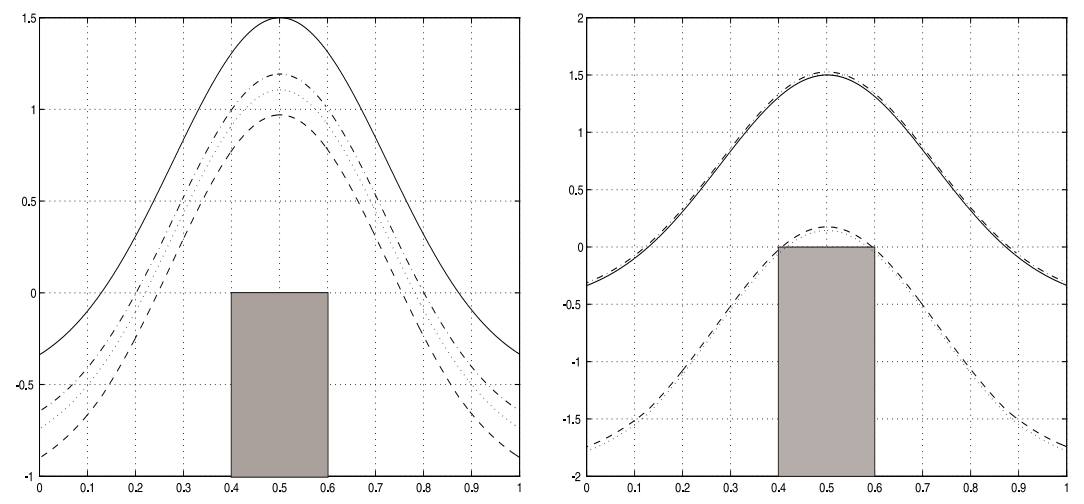

FIG. 4.1. The functions $\phi^{(0)}$ (solid line), $\phi^{(1)}$ (dashed line), $\phi^{(2)}$ (dash-dot line) and $\phi^{(3)}$ (dotted line). To recover is the interval [0.4,0.6], which is displayed by the grey rectangle. The first iteration is the best. In the right picture $\alpha$ is smaller than in the left picture. 


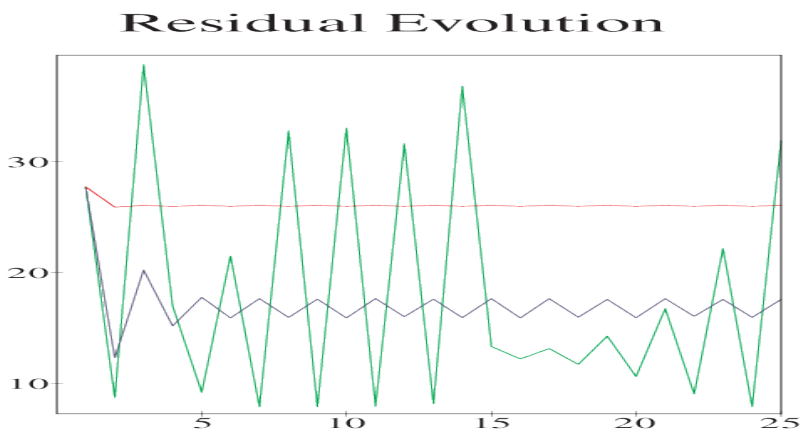

FIG. 4.2. Decay of the residual $\left\|F\left(P_{\varepsilon}\left(\phi^{(k)}\right)\right)-y^{\delta}\right\|_{Y}^{2}$ in dependence of the number of iterations (residual evaluated for the first experiment - noise free data in Section 5). After the first iteration the fixed-point iteration stagnates.

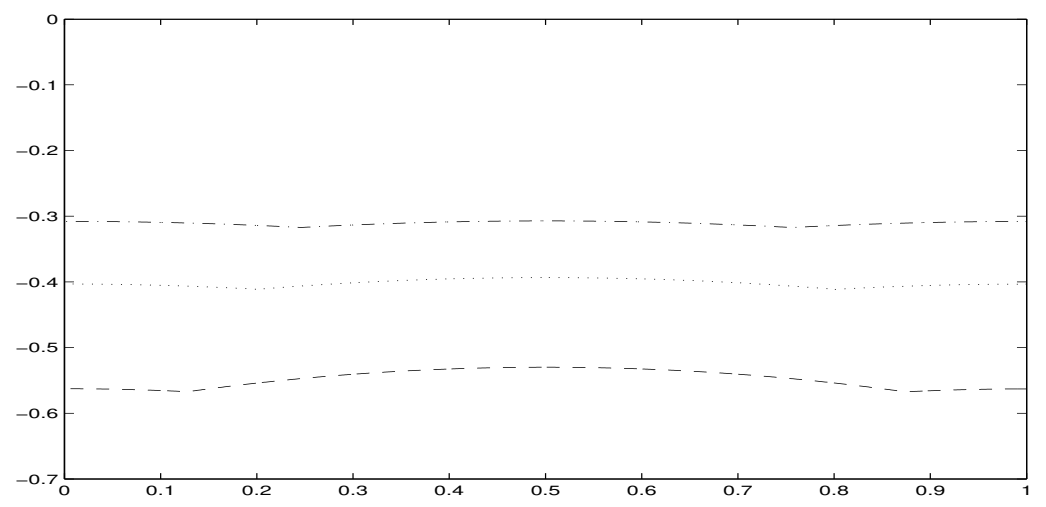

FIG. 4.3. The differences between $\phi^{(0)}$ and the functions $\phi^{(1)}$ (dashed line), $\phi^{(2)}$ (dash-dot line) and $\phi^{(3)}$ (dotted line) from the left picture of figure 4.1.

5. Numerical Experiments. In this section we shall consider an inverse potential problem of recovering the shape of a domain $D$ using the knowledge of its (constant) density and the measurements of the Cauchy data of the corresponding potential on the boundary of a fixed Lipschitz domain $\Omega \subset \mathbb{R}^{2}$, which contains $\bar{D}$. This is the same problem as considered by Hettlich and Rundell [13, which used iterative methods for recovering a single star-shaped object.

To achieve an analogous problem, a certain definition of the operator $F$ is necessary:

$$
\begin{aligned}
F: L^{2}(\Omega) & \rightarrow L^{2}(\partial \Omega) \\
\chi_{D} & \rightarrow F\left(\chi_{D}\right)
\end{aligned}
$$

This is possible, because we consider only characteristic functions $\chi_{D}$. The $L^{2}(\Omega)$ norm is then equivalent to the $L^{1}(\Omega)$-norm of $\chi_{D}$. Therefore the necessary properties are retained.

The problem introduced above can mathematically be described as follows:

$$
\Delta u=\chi_{D}, \text { in } \Omega ;\left.u\right|_{\partial \Omega}=0,
$$


where $\chi_{D}$ is the characteristic function of the domain $D \subset \Omega$, which has to be reconstructed. Since $\chi_{D} \in L^{2}(\Omega)$, the Dirichlet boundary value problem in 5.1 has a unique solution, the potential $u \in H^{2}(\Omega) \cap H_{0}^{1}(\Omega)$. Here $H_{0}^{1}(\Omega)$ is defined as the closure with respect to $H^{1}(\Omega)$ of functions in $C^{\infty}(\Omega)$ with compact support in $\Omega$.

The inverse problem we are concerned with, consists in determining the shape of $D$ from measurements of the Neumann trace of $u$ at $\partial \Omega$, i.e. from $[\partial u / \partial \nu]_{\partial \Omega}$, where $\nu$ represents the outer normal vector to $\partial \Omega$.

Notice that this problem can be considered in the framework of an inverse problem for the Dirichlet to Neumann map. For given $h \in L^{2}(\Omega)$, the Dirichlet to Neumann operator maps a Dirichlet boundary data onto the Neumann trace of the potential, i.e., $\Lambda: H^{1 / 2}(\partial \Omega) \rightarrow H^{-1 / 2}(\partial \Omega), \Lambda(\varphi):=[\partial \tilde{u} / \partial \nu]_{\partial \Omega}$, where $\tilde{u}$ solves

$$
\Delta \tilde{u}=h, \text { in } \Omega ;\left.\quad \tilde{u}\right|_{\partial \Omega}=\varphi .
$$

The inverse problem for the $\Lambda$ operator consists in determining the unknown parameter (i.e., the function $h$ ) from different pairs of Dirichlet, Neumann boundary data. The general case with $h \in L^{2}(\Omega)$ has already been considered by many authors, among them we mention [7, 24, which introduced numerical methods based on Tikhonov regularization, and [13] with iterative regularization methods.

Hettlich and Rundell [13] observe that, in the particular case $h=\chi_{D}$, one pair of measurement data of Dirichlet-Neumann data furnishes as many information as the full Dirichlet-Neumann operator, i.e., it is sufficient to consider only one pair of Cauchy data for the inverse problem. Therefore, no further information on $D$ can be gained by using various pairs of Dirichlet-Neumann data, since we can always reduce the reconstruction problem to the homogeneous Dirichlet case.

For the particular case $h=\chi_{D}$, it has been observed by Hettlich and Rundell [13] that the Cauchy data may not furnish enough information to reconstruct the boundary of $D$, e.g., if $D$ is not simply connected. On the other hand, Isakov observed in [14] that star like domains $D$ are uniquely determined by their potentials.

The inverse potential problem is discussed within the general framework introduced in Section 1. In particular, we allow domains, that consists of a number of connected inclusions. For this general class we have not unique identifiability and we restrict attention to "minimum-norm solutions". Recall that in this case a minimumnorm-solution is a level set function $\phi$, where $P(\phi)$ determines the inclusion. A minimum norm solution satisfies that it minimizes the functional $\rho(z, \phi)$ in the class of level set functions such that the according Neumann boundary values $\frac{\partial u}{\partial \nu}$ fit the data $y^{\delta}$.

5.1. The level set regularization algorithm. In the following we describe the level set regularization algorithm. This method compares to the Landweber iteration as proposed by Hettlich and Rundell [13]. In our context the operator $F^{\prime}$ can be considered as an approximation of the domain derivative operator for multiple connected domains (cf. Figure 5.1).

The complexity of our algorithm is as follows: at each iteration of the level set method, three elliptic boundary value problems are solved (two of Dirichlet type and one of Neumann type).

In Table 5.1 the iteration procedure for the solution of the formal optimality condition 3.2 is outlined. The algorithm can be implemented using finite element codes (as we did) or finite difference methods for the solution of partial differential equations. 
1. Evaluate the residual $r_{k}:=F\left(P_{\varepsilon}\left(\phi_{k}\right)\right)-y^{\delta}=\frac{\partial u_{k}}{\partial \nu}-y^{\delta}$, where $u_{k}$ solves

$$
\Delta u_{k}=P_{\varepsilon}\left(\phi_{k}\right), \text { in } \Omega ;\left.\quad u_{k}\right|_{\partial \Omega}=0 .
$$

2. Evaluate $v_{k}:=F^{\prime}\left(P_{\varepsilon}\left(\phi_{k}\right)\right)^{*}\left(r_{k}\right) \in L^{2}(\Omega)$, solving

$$
\Delta v_{k}=0, \text { in } \Omega ;\left.\quad v_{k}\right|_{\partial \Omega}=r_{k} .
$$

3. Evaluate $w_{k} \in H^{1}(\Omega)$, satisfying

$$
\begin{aligned}
(I-\Delta) w_{k} & =-P_{\varepsilon}^{\prime}\left(\phi_{k}\right) v_{k}+\beta \alpha P_{\varepsilon}^{\prime}\left(\phi_{k}\right) \nabla \cdot\left(\frac{\nabla P_{\varepsilon}\left(\phi_{k}\right)}{\left|\nabla P_{\varepsilon}\left(\phi_{k}\right)\right|}\right), \text { in } \Omega ; \\
\left.\frac{\partial w_{k}}{\partial \nu}\right|_{\partial \Omega} & =0 .
\end{aligned}
$$

4. Update the level set function $\phi_{k+1}=\phi_{k}+\frac{1}{\alpha} w_{k}$.

FIG. 5.1. Implementation of a single iteration step for minimizing the level set regularization.

5.2. Reconstruction of a density function with non simply connected support. In this first experiment we consider the inverse problem of reconstructing the right hand side $\chi_{D}$ in (5.1) from the knowledge of a single pair of boundary data $(u, \Lambda u)=\left(0, y^{\delta}\right)$ at $\partial \Omega$. In the examples considered below we always use the squared domain $\Omega=(0,1)^{2} \subset \mathbb{R}^{2} \cdot \chi_{D} \in L^{2}(\Omega)$ is the characteristic function as represented in Figure 5.2

The overdetermined boundary measurement data $y^{\delta}$ for solving the inverse problem, is obtained by solving the elliptic boundary value problem in (5.1). Notice that $\chi_{D}$ corresponds to the characteristic function of a not-connected proper subset of $\Omega$. The initial condition for the level set function is shown in Figure 5.2. In order to
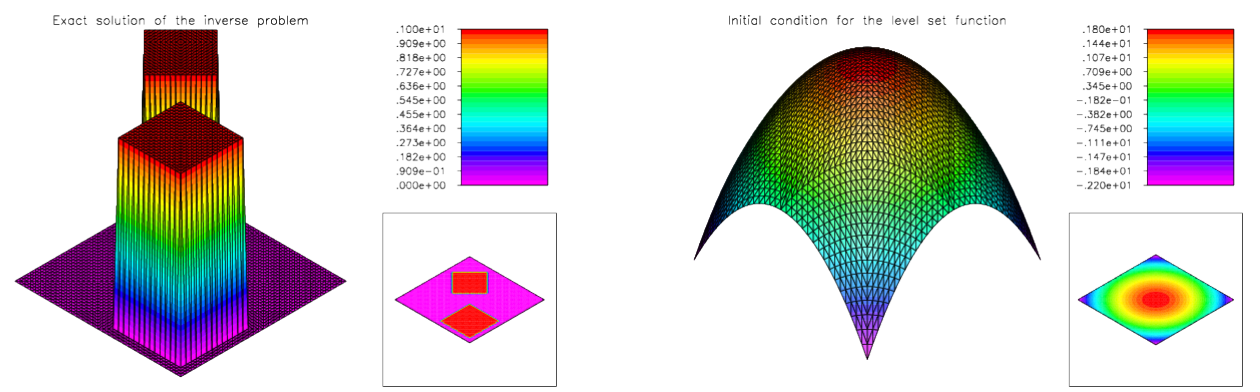

FIG. 5.2. The picture on the left hand side shows the coefficient to be reconstructed. On the other picture, the initial condition for the level set regularization method.

avoid inverse crimes, the direct problem (5.1) is solved on an adaptively refined grid with 8.807 nodes (three levels of adaptive refinement). Alternatively, in the numerical implementation of the level set method, all boundary value problems are solved at an uniformly refined grid with 2.113 nodes.

When the data is given exactly, we tested the iterative level set regularization without the additional regularization term $\left|P_{\varepsilon}\left(\phi_{k}\right)\right|_{\mathrm{BV}}$, i.e. $\beta=0$. 
In all computed experiments we use the operator $P_{\varepsilon}$ defined in Section 2 with $\varepsilon=1 / 8$. This seams to be compatible with the size of our mesh, since the diameter of the triangles in the uniform grid (used in the finite element method) is approximately $\sqrt{2} / 32$.

In Figure 5.3 we present the evolution of the level set function for given exact data for the first 3000 iterative steps. As one can see in this figure, the original level set splits into two convex components after approximately 800 iterations. After 1000 iterations the level set function still changes, but very slowly. We performed similar tests for different initial conditions and observed that, after 1000 iterations, the corresponding pictures look very much alike.

For the second part of this experiment, the density function to be reconstructed is still the one shown in Figure 5.2. This time, however, we add randomly generated noise to the data $y^{\delta}$ used in the first part of the experiment.

The exact boundary data $y^{\delta}$ is shown in Figure 5.4 as the dotted (blue) line. We consider actually two distinct sets of perturbed data: For the first experiment we add to the exact data a white noise of $10 \%$ (in the $l_{\infty}$-norm); For the second experiment we use a noise level of 50\%. Both sets of inaccurate data are plotted in Figure 5.4 and correspond to the solid (red) line.

As in the noise free experiment, the same care was taken to avoid inverse crimes. The choice of the parameter $\varepsilon$ (operator $P_{\varepsilon}$ ) follows also the same criteria as before. However, since we are now dealing with noisy data, we have to develop a strategy for the choice of the regularization parameter $\beta$. For this proposal we opted for the fitto-data strategy, i.e. $\beta \alpha$ is chosen such that the regularization term (see Figure 5.1) has the same order as the noise level.

The corresponding results generated by the level set method where surprisingly stable, as one can observe in Figures 5.5 and 5.6. In the first case (noise level of $10 \%$ ) the results are comparable with the previous experiment, where exact data was available. In the second case (noise level of 50\%) we are not able to precisely recover the shape of the set $D$, corresponding to the characteristic function shown in Figure 5.2. However, we are still able to identify the number of connected components of $D$, as well as their relative positions inside the domain $\Omega$.

5.3. Reconstruction of a density function with non convex support. In this second experiment we consider the problem of reconstructing the density function shown in Figure 5.7. The main goal now is to investigate the difficulty of the level set method in recovering non convex domains. The domain $\Omega$ is the same used in Subsection 5.2 and again we aim to reconstruct the density function in (5.1) from boundary measurements.

As in the first part of the previous experiment, the data is almost given exactly and the velocity $w_{k}$ is again obtained by solving the boundary value problem with $\beta=0$. The evolution of the level set function is shown in Figure 5.8 .

REMARK 5.1. The effect of parameter changes: In our numerical observations we observed that in numerical simulations the minimizer is not severely affected by the choice of $\beta \alpha$ and can in fact be neglected.

Acknowledgment. The work of F.F. has been supported by the Tiroler Zukunftsstiftung; the work of O.S. has been partly supported by the FWF (Austrian Science Foundation), grant Y-123 INF. A.L. is on leave from Department of Mathematics, Federal Univ. of St. Catarina, Brazil; his work is supported by the Austrian Academy of Sciences and CNPq, grant 305823/2003-5. 

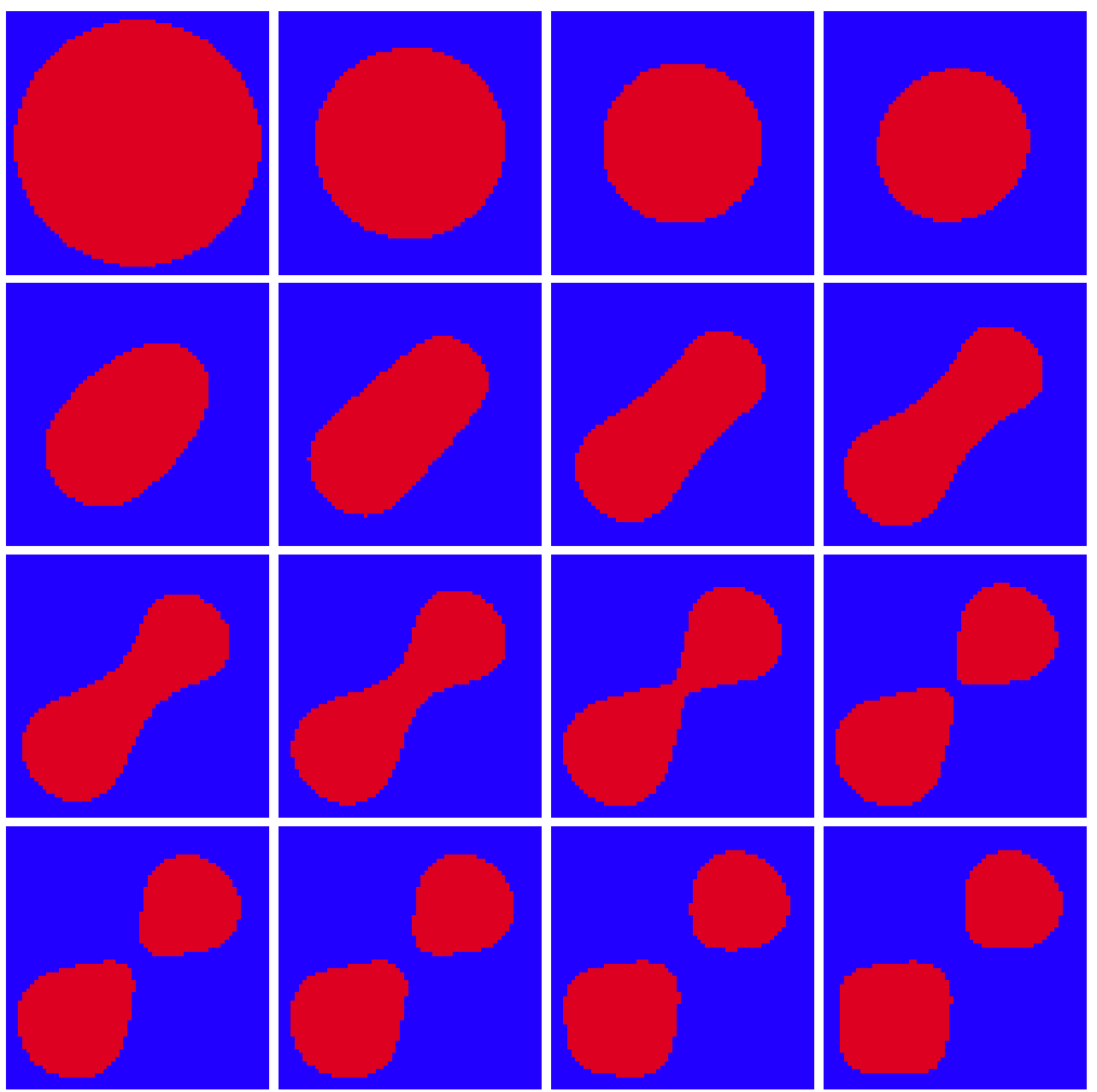

Fig. 5.3. Level set evolution for exact data. Plots after 0,1,2,10, 100,200,300,400, 500,600,700,800, 900,1000, 2000, 3000 iterative steps.

\section{REFERENCES}

[1] R. Acar and C.R. Vogel. Analysis of bounded variation penalty methods for ill-posed problems. Inverse Probl., 10:1217-1229, 1994.

[2] R.A. Adams. Sobolev Spaces. Academic Press, New York, 1975.

[3] L. Ambrosio. Geometric evolution problems, distance function and viscosity solutions. In [4], pages 5-94, 1999.

[4] L. Ambrosio and N. Dancer. Calculus of Variations and Partial Differential Equations. Springer, 1999.

[5] H. Brezis. Operateurs Maximaux Monotones et semi-groupes de contractions dans les espaces de Hilbert. North-Holland, Amsterdam, 1973.

[6] M. Burger. A level set method for inverse problems. Inverse Problems, 17(5):1327-1355, 2001.

[7] H. Cabayan and G. Belford. On computing a stable least squares solution to the inverse problem for a planar Newtonian potential. SIAM J. Appl. Math., 20:51-61, 1971.

[8] T. Chan, J. Shen, and L. Vese. Variational PDE models in image processing. Notices Amer. Math. Soc., 50:14-26, 2003.

[9] S. Chen, B. Merriman, S. Osher, and P. Smereka. A simple level set method for solving Stefan problems. J. Comput. Phys., 135:8-29, 1997. 

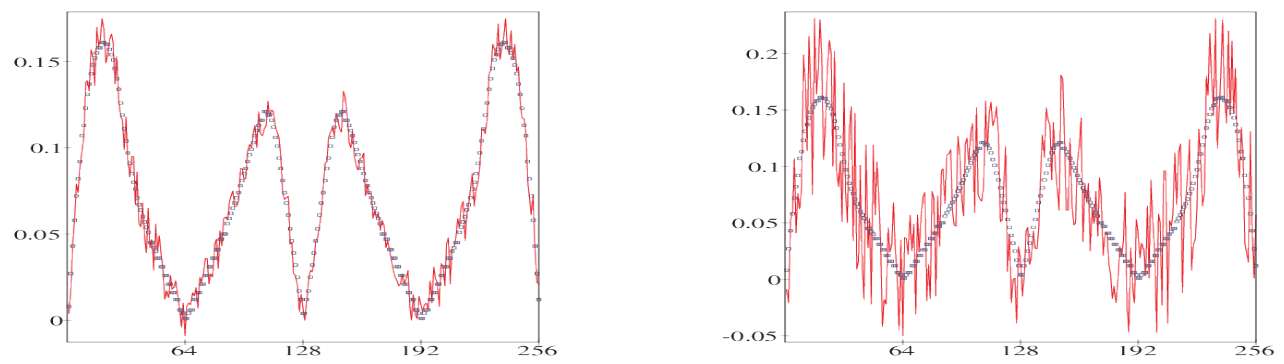

FIG. 5.4. The dotted (blue) line represents the exact data $y^{\delta}$; the solid (red) line represents the perturbed data. The noise level corresponds to $10 \%$ at the left hand side and $50 \%$ at the right hand side.

[10] M. Delfour, and J. -P. Zolesio. Shape analysis via oriented distance functions. Journal of Functional Analysis, 123:129-201, 1994.

[11] H.W. Engl, M. Hanke, and A. Neubauer. Regularization of Inverse Problems. Kluwer Academic Publishers, Dordrecht, 1996.

[12] H.W. Engl, K. Kunisch, and A. Neubauer. Convergence rates for Tikhonov regularization of nonlinear ill-posed problems. Inverse Probl., 5:523-540, 1989.

[13] F. Hettlich and W. Rundell. Iterative methods for the reconstruction of an inverse potential problem. Inverse Probl., 12:251-266, 1996.

[14] V. Isakov. Inverse Source Problems. American Mathematical Society, Providence, Rhode Island, 1990.

[15] R. Kohn and G. Strang. Optimal design and relaxation of variational problems. I. Comm. Pure Appl. Math., 39:113-137, 1986.

[16] R. Kohn and G. Strang. Optimal design and relaxation of variational problems. II. Comm. Pure Appl. Math., 39:139-182, 1986.

[17] R. Kohn and G. Strang. Optimal design and relaxation of variational problems. III. Comm. Pure Appl. Math., 39:353-377, 1986.

[18] A. Leitão and O. Scherzer. On the relation between constraint regularization, level sets, and shape optimization. Inverse Problems, 19:L1-L11, 2003.

[19] B. Merriman, J. Bence, and S. Osher. Motion of multiple functions: a level set approach. J. Comput. Phys., 112:334-363, 1994.

[20] V.A. Morozov. Methods for Solving Incorrectly Posed Problems. Springer Verlag, New York, Berlin, Heidelberg, 1984.

[21] V.A. Morozov. Regularization Methods for Ill-Posed Problems. CRC Press, Boca Raton, 1993.

[22] M.Z. Nashed and O. Scherzer. Least squares and bounded variation regularization with non differentiable functional. Num. Funct. Anal. and Optimiz., 19:873-901, 1998.

[23] S. Osher and J. A. Sethian. Fronts propagating with curvature-dependent speed: Algorithms based on Hamilton-Jacobi formulations. J. Comput. Phys., 79:12-49, 1988.

[24] W. Ring. Identification of a core from boundary data. SIAM J. Appl. Math., 55:677-706, 1995.

[25] F. Santosa. A level-set approach for inverse problems involving obstacles. ESAIM Contrôle Optim. Calc. Var., 1:17-33 (electronic), 1995/96.

[26] T.I. Seidman and C.R. Vogel. Well posedness and convergence of some regularization methods for non-linear ill posed problems. Inverse Probl., 5:227-238, 1989.

[27] Hong-Kai Zhao, T. Chan, B. Merriman, and S. Osher. A variational level set approach to multiphase motion. J. Comput. Phys., 127:179-195, 1996. 

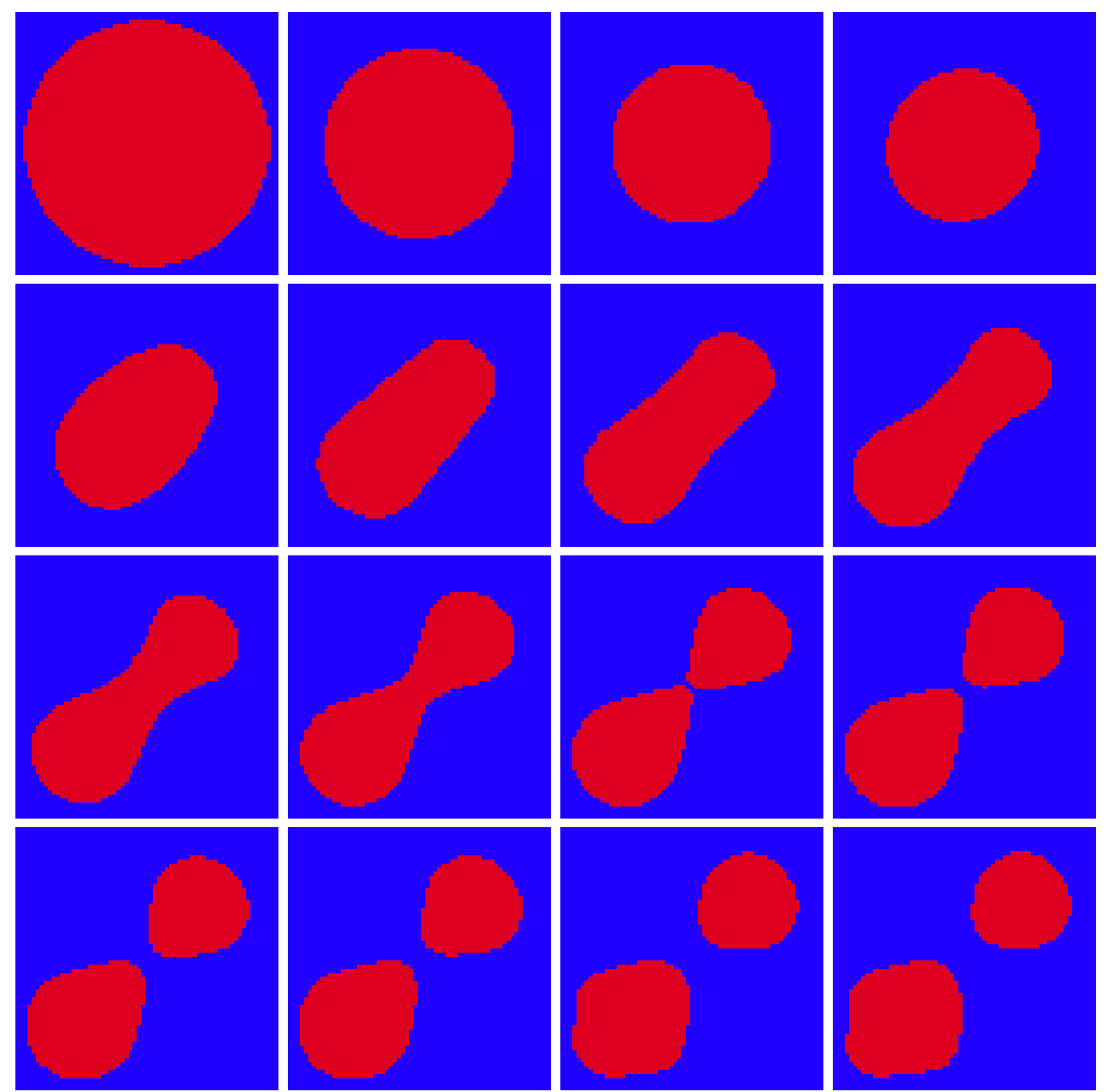

FIG. 5.5. Level set evolution for inaccurate data; noise level of $10 \%$. Plots after $0,1,2,10$, 100, 200,300, 400, 500,600,700,800, 900, 1000, 2000, 3000 iterative steps. 

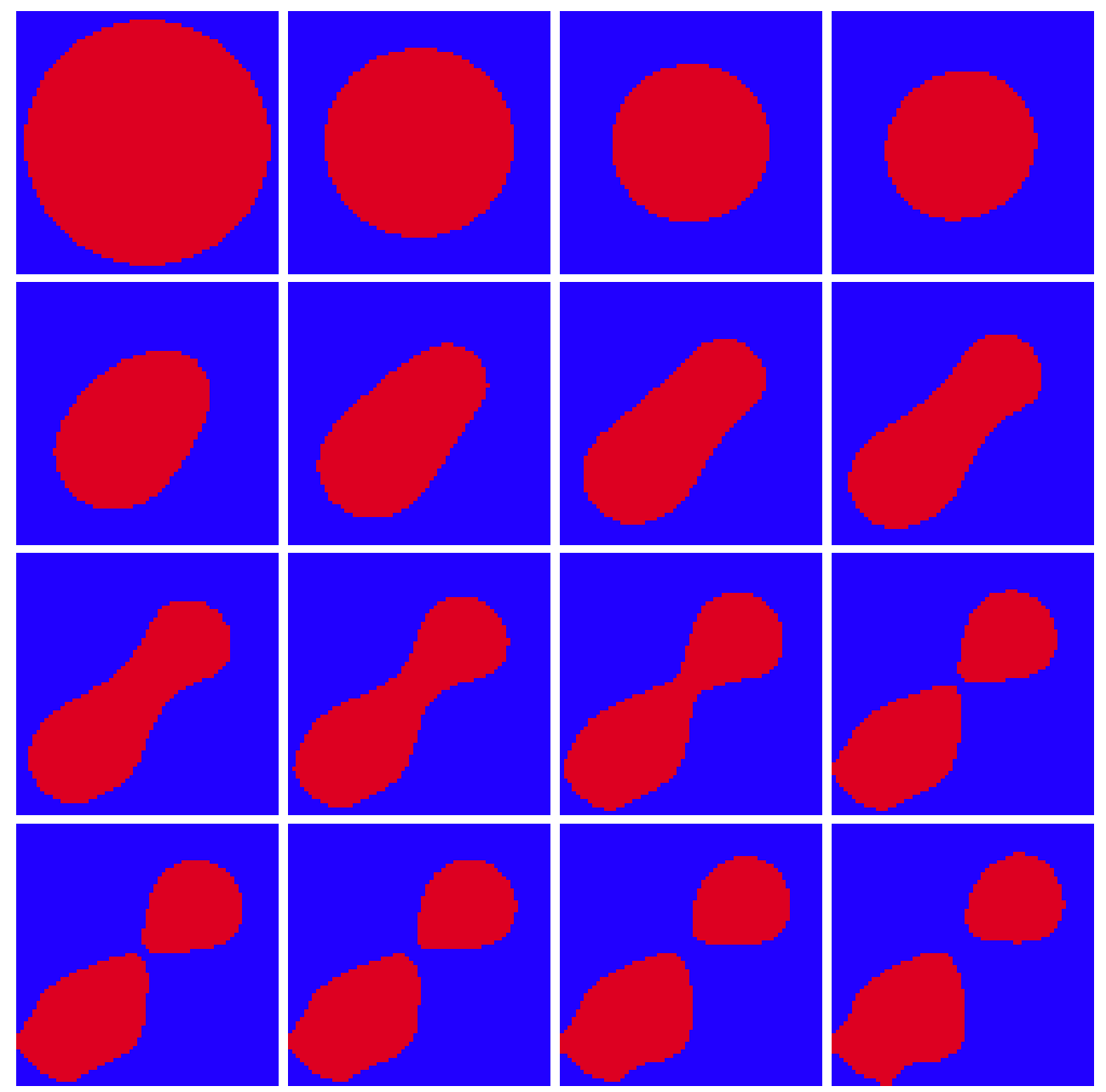

Fig. 5.6. Level set evolution for inaccurate data; noise level of 50\%. Plots after $0,1,2,10$, 100, 200,300,400, 500,600,700,800, 900,1000,1300, 1600 iterative steps.
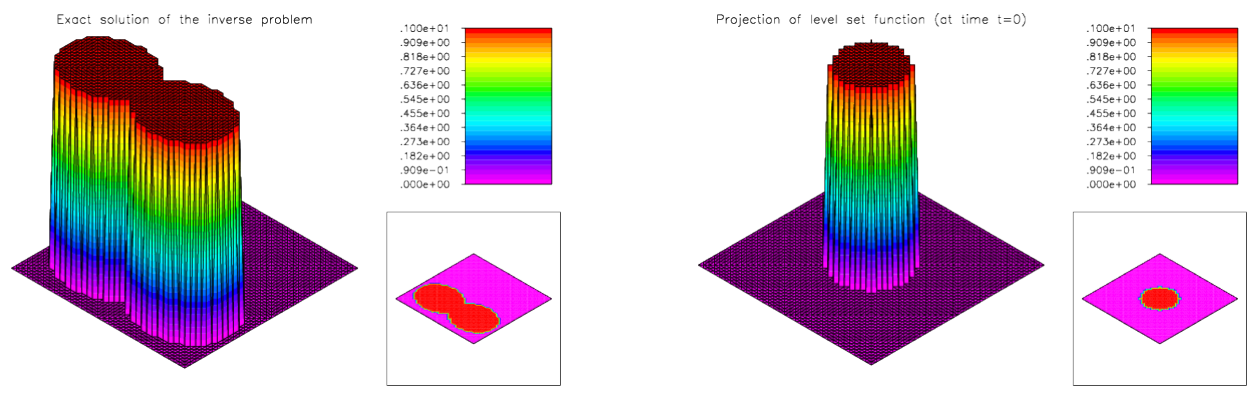

FIG. 5.7. The picture on the left hand side shows the coefficient to be reconstructed. On the other picture, the (projection of the) initial condition for the level set regularization method. 

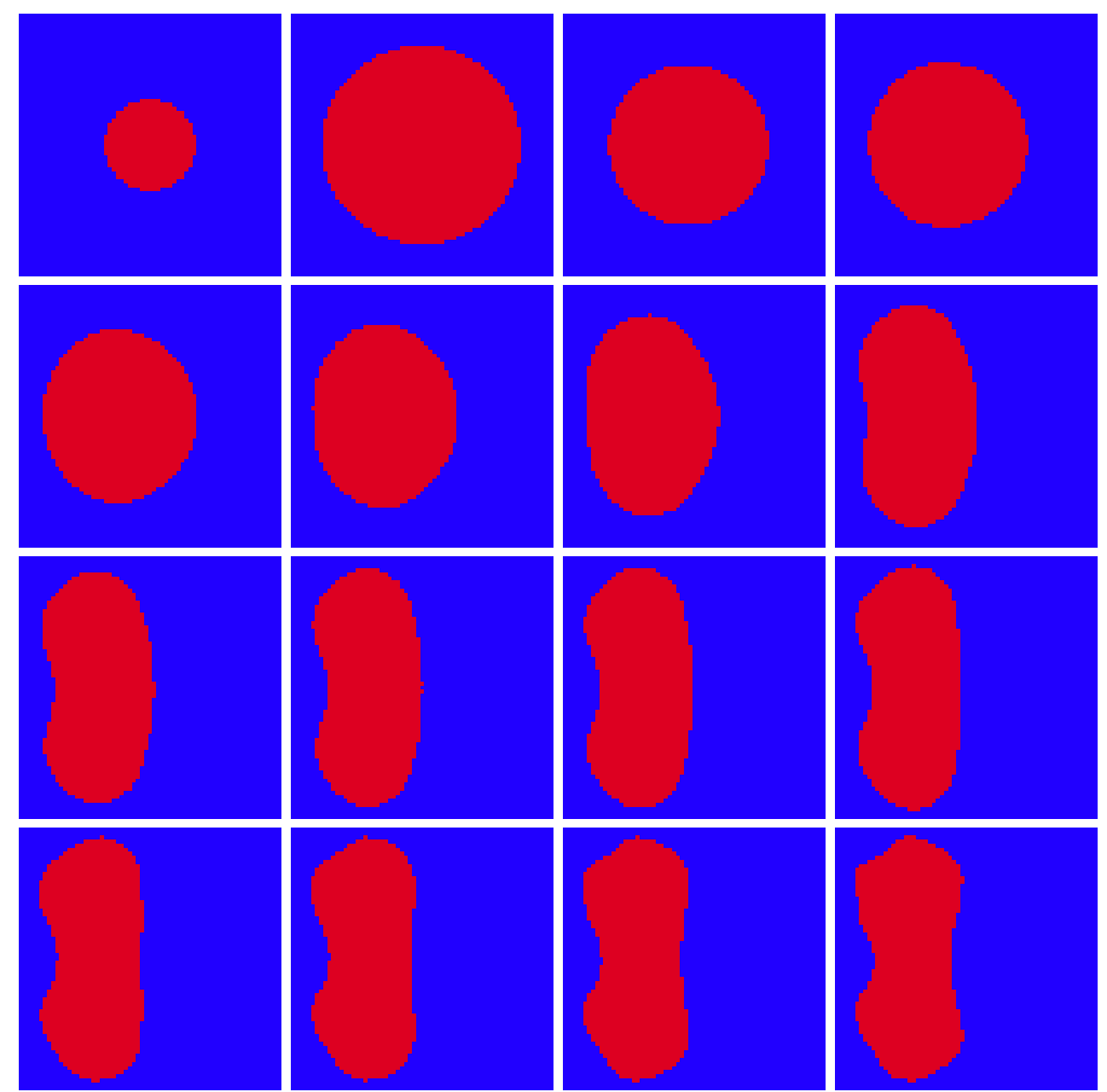

Fig. 5.8. Level set evolution for second experiment. Plots after 0,1,5, 20, 50,100, 200, 400, $600,800,1000,2000,5000,10000,20000,50000$ iterative steps. 